\title{
Characterisation of the normal right ventricular pressure-volume relation by biplane angiography and simultaneous micromanometer pressure
}

\author{
measurements
}

\author{
ANDREW N REDINGTON, HUON H GRAY, MARGARET E HODSON, \\ MICHAEL L RIGBY, PAUL J OLDERSHAW
}

From the Cardiac Department, Brompton Hospital, London

SUMMARY The normal right ventricular pressure-volume relation was studied by recording biplane right ventriculograms with simultaneous high fidelity pressure recordings in 10 adults found to have normal coronary arteries and haemodynamic function at diagnostic cardiac catheterisation. Right ventricular volume was measured frame by frame from digitised ventriculograms by a modification of Simpson's rule. The accuracy of this method was tested in a study of 22 human and animal right ventricular casts. There was excellent agreement between calculated volumes and those measured by fluid displacement. The derived regression equations were used to correct right ventricular volumes calculated from in vivo studies. The mean (SD) end diastolic volume index for the group was $62(13) \mathrm{ml} / \mathrm{m}^{2}$, the stroke volume index was $43(8) \mathrm{ml} / \mathrm{m}^{2}$, and the ejection fraction was $62(6) \%$. Right ventricular pressure-volume loops were generated by combining simultaneous volume and pressure curves. The normal right ventricular pressure-volume loop was triangular, departing significantly from the square or rectangle of the normal left ventricular pressure-volume loop. Ejection from the right ventricle began early during the pressure rise and continued as right ventricular pressure fell. As a result phases of isovolumic contraction and relaxation were difficult to define.

These observations show that normal right ventricular pressure-volume relations differ considerably from those of the normal left ventricle, presumably reflecting the different loading conditions of the two ventricles.

Unlike the left ventricle, the right ventricle has been largely ignored in terms of the detailed characterisation of its function. Probably the most important factor responsible for this has been the difficulty in accurately measuring right ventricular volume as a result of its complex geometric shape. Unlike the normal left ventricle, which approximates to a prolate ellipsoid, the right ventricle does not lend itself to volume calculation on the basis of a simple geometric assumption. None the less, various methods

Requests for reprints to Dr Andrew $\mathrm{N}$ Redington, Cardiac Department, Brompton Hospital, Fulham Road, London SW3 6HP.

Accepted for publication 14 July 1987 have been used, including simple area-length algorithms and geometric models ${ }^{1-3}$ and the use of Simpson's rule, which permits the measurement of the volume of a complex shape by dividing it into a series of slices. ${ }^{245}$ Although they require computer techniques to make such measurements practicable, methods incorporating Simpson's rule can be applied to the determination of right ventricular volume both throughout the cardiac cycle and as it is affected by disease.

Although the importance of right ventricular dysfunction in the pathogenesis of ischaemic, ${ }^{6}$ and congenital heart disease ${ }^{7}$ has been emphasised, most previous studies have been restricted to simple measurements of right ventricular volumes and ejection fraction. Few attempts have been made to apply to 
the right ventricle those more sophisticated indices of function that have greatly enhanced our knowledge of the left ventricle, such as data derived from ventricular pressure-volume loops. An animal study $^{8}$ and a radionuclide study in patients with acquired heart disease ${ }^{9}$ have suggested that there may be fundamental differences between the pressure-volume relations of the normal left and right ventricles; however, the normal right ventricular pressure-volume relation has yet to be defined angiographically. This study was therefore designed to examine the normal right ventricular pressurevolume relation, using Simpson's rule to measure right ventricular volume from biplane right ventriculograms combined with simultaneous high fidelity pressure recordings.

\section{Patients and methods}

\section{RIGHT VENTRICULAR VOLUME \\ DETERMINATION \\ Right ventricular casts}

Twenty two radio-opaque casts of animal (ovine, porcine $n=16)$, and human $(n=6)$ right ventricles were made with acrylic resin and radiographic contrast material. The hearts were prepared by oversewing both atrioventricular valves, and a purse string suture (placed around each great vessel) was tightened around a large bore cannula through which the resin-contrast mixture was introduced. The right and left ventricles were distended to filling pressures of 10 and $13 \mathrm{~cm}$ of resin and contrast mixture (equivalent to approximately 8 and $10 \mathrm{~mm} \mathrm{Hg}$ respectively) in an attempt to preserve the normal geometry of the interventricular septum. The hardened cast was dissected from the right ventricle and any residual tissue was removed by immersion in concentrated sodium hydroxide. Cast volumes were determined by water displacement and the mean of five separate estimations was taken.

\section{Angiographic volume estimation}

Each cast was then placed in its anatomical position on the catheterisation table. Biplane ventriculograms were recorded on $35 \mathrm{~mm}$ cinefilm at 50 frames per second in anteroposterior, lateral, and $30^{\circ}$ right anterior oblique, $60^{\circ}$ left anterior oblique projections. Calibration was made by a modification of the linear shift method, ${ }^{10}$ and the magnification factor was calculated from angiograms exposed before and after the cast was moved $10 \mathrm{~cm}$ within the $x$ ray field such that:

$$
\text { magnification factor }=\text { observed shift/actual shift. }
$$

A single frame from each of the orthogonal views was then projected on to a Summagraphics digi- tising plate interfaced with a Prime 750 computer and the cast outline was traced by hand. No attempt was made to follow precisely the pattern of trabeculae or papillary muscles. Instead a smooth line was traced around the outermost perimeter of the cast silhouette. For each outline approximately 100 $x, y$ coordinates were recorded and stored on magnetic tape for later analysis. Three dimensional reconstructions were plotted from these stored data (fig 1) by a computer program that is described in detail elsewhere. ${ }^{11}$ We used Simpson's rule to calculate the volume of the casts. Briefly, the ventricular outline was divided into 40 equal segments along its long axis. The cross sectional area of each segment was assumed to be elliptical and the diameters of the major and minor axes were calculated from each of the angiographic views. Simpson's rule was then applied to calculate the volume of each slice, and the total volume of the ventricle according to the formula:

Volume $=\frac{\pi \mathrm{h}}{3}\left(\sum_{1}^{39} \mathrm{~d} 1_{\mathrm{O}} \mathrm{d} 2_{\mathrm{O}}+1 / 2 \sum_{2}^{40} \mathrm{~d} 1_{\mathrm{E}} \mathrm{d} 2_{\mathrm{E}}\right)$,

where $\mathrm{h}=$ slice depth, $\mathrm{d} 1$ and $\mathrm{d} 2$ are the major and minor diameters respectively, and $\mathrm{O}=$ odd numbered slices, and $\mathrm{E}=$ even numbered slices.

\section{PATIENTS}

Ten adults (mean age 37 years, range 29-47, six men, four women) were studied. All were undergoing cardiac catheterisation to establish a clear diagnosis for atypical chest pain. No patient had clinical or electrocardiographic evidence of previous myocardial infarction or arrhythmia and no patient was taking regular cardiac medication. The Brompton Hospital Ethical Committee approved the study and all patients gave their informed written consent.

\section{Cardiac catheterisation}

Patients were studied without premedication. Routine left ventriculography and coronary angiography were performed from a femoral approach with the patient supine. After the demonstration of normal left heart haemodynamic function, left ventriculography, and coronary arteriography at least 15 minutes were allowed to elapse before right heart catheterisation was performed. All right heart pressures were measured by a micromanometer tipped catheter with a high-flow lumen through which angiography was performed (Millar Instruments Inc). The transducer of this catheter has a linear response with an output accuracy of $\pm 0.5 \%$ between -50 and $+300 \mathrm{~mm} \mathrm{Hg}$ and a frequency response from 0 to $20 \mathrm{kHz}$. Before insertion the transducer was set at zero for the mid-chest level and calibrated to $+100 \mathrm{~mm} \mathrm{Hg}$ with an external cali- 


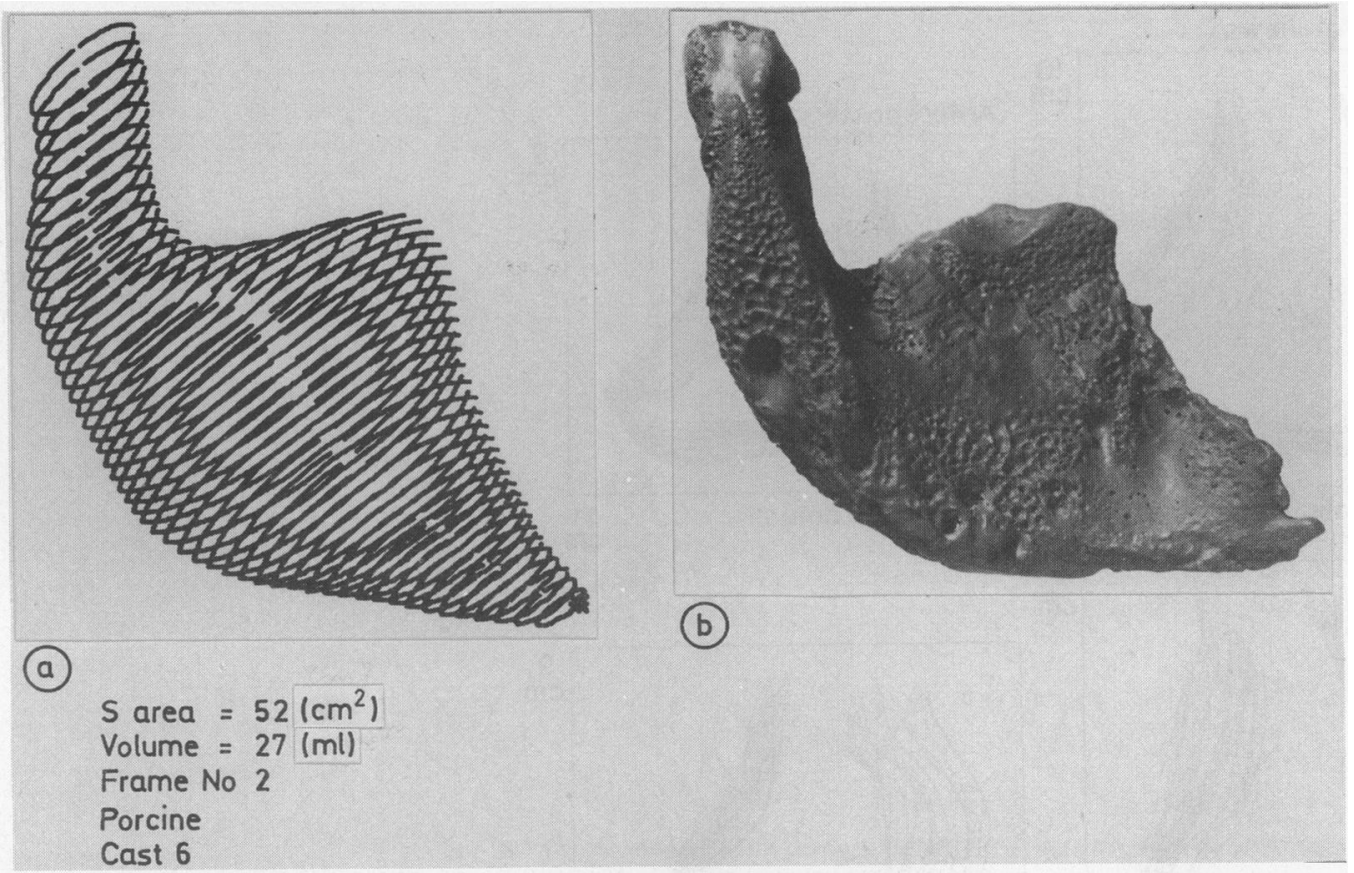

Fig 1 Right ventricular cast and computer-derived three dimensional reconstruction from biplane angiogram.

bration source. We took care to position the catheter tip in the apex of the right ventricle. While the patients held their breath mid-inspiration we recorded biplane right ventriculograms in anteroposterior and lateral projections on $35 \mathrm{~mm}$ film during the injection of $30 \mathrm{ml}$ of radiographic contrast medium (Isopaque). Simultaneous recordings of right ventricular pressure were made at a paper speed of $200 \mathrm{~mm} / \mathrm{s}$ and an event mark was pressed when contrast material appeared in the right ventricle. To enable exact synchronisation an electrocardiograph timing mark was recorded simultaneously on both cine and pressure recordings. At the end of the study the catheter was left in the right ventricle and the patient was shifted $10 \mathrm{~cm}$ in a craniocaudal direction. A cine film was recorded in both projections for later calibration.

\section{ANALYSIS OF ANGIOGRAMS}

Each view of the biplane right ventriculogram was digitised with the equipment described above. Only sinus beats were analysed; extrasystoles and postextrasystoles were discarded. Starting at the $R$ wave of the electrocardiogram successive frames were projected on to the digitising table and cavity outlines were traced frame by frame throughout the cycle. Once again no attempt was made to follow the trabecular pattern precisely. This procedure was repeated for the same cycle in the orthogonal view and finally the pressure curve recorded during the cycle was digitised by the same equipment, data points being extracted every $20 \mathrm{~ms}$ corresponding to each frame of the angiogram. All data were stored on magnetic tape and the following were derived:

(a) Superimposed plots of cavity outlines throughout the cardiac cycle (fig 2)

(b) Graphs displaying changes of cavity volume, and pressure throughout the cardiac cycle (fig 3), from which measurements of end diastolic and end systolic volumes were made and corrected for body surface area $\left(\mathrm{ml} / \mathrm{m}^{2}\right)$. Right ventricular ejection fraction was calculated as the ratio of stroke volume to end diastolic volume.

(c) Pressure-volume loops showing instantaneous changes of pressure and volume throughout the cardiac cycle (figs 4 and 5 ), and from these loops the following measurements were made: $(i)$ right ventricular ejection before and after peak right ventricular pressure expressed as a percentage of the total stroke volume; (ii) the external mechanical work performed by the right ventricle during systole. This is equivalent to the area enclosed by the pressure volume loop and was determined by automatic planimetry, with systolic stroke work being expressed in $\mathrm{J} / \mathrm{m}^{2}$ body surface area $(1 \mathrm{~mm} \mathrm{Hg} \cdot \mathrm{ml}=$ $1.36 \times 10^{-4} \mathrm{~J}$ ); and (iii) cycle efficiency, which was 
Fig 2 Superimposed plots of cavity outlines from successive frames of each of the biplane views of a right ventriculogram.

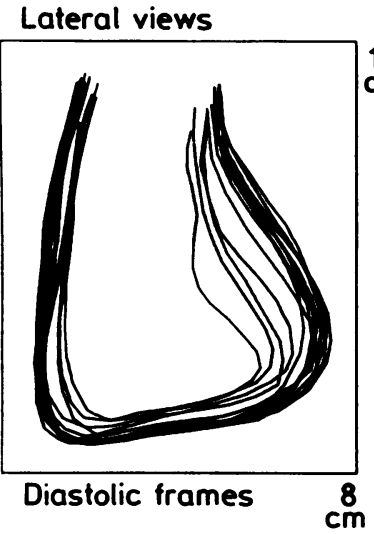

cm

Anteroposterior views
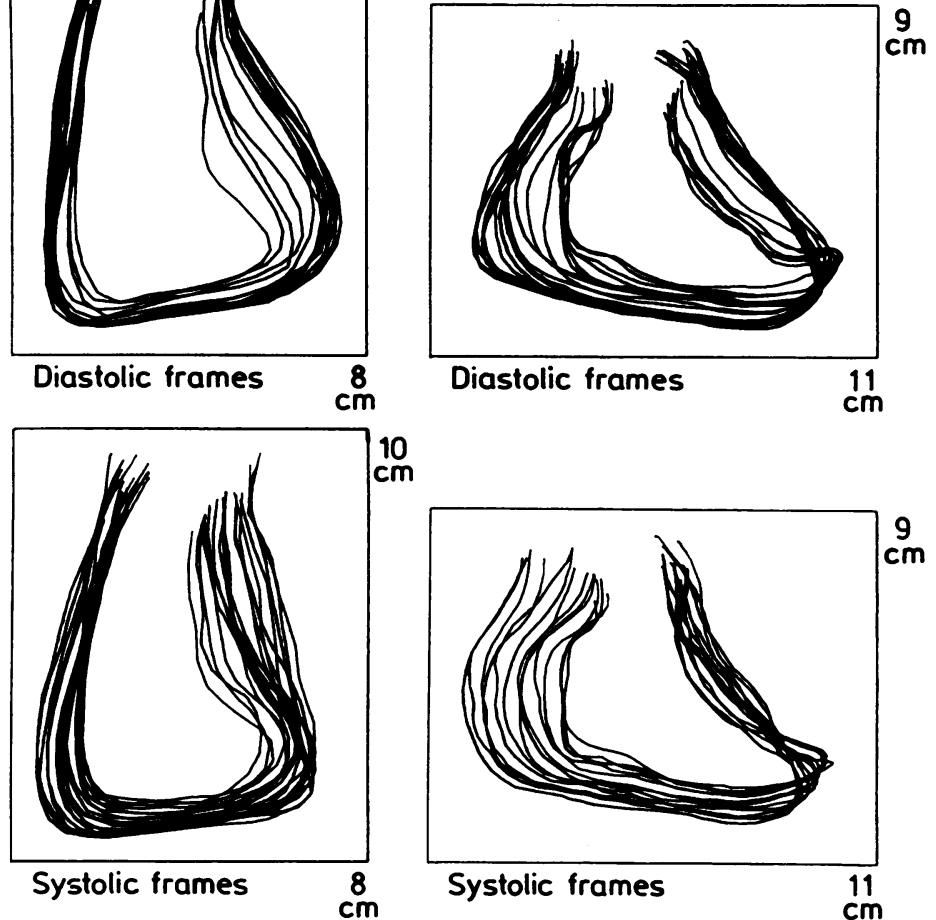

10
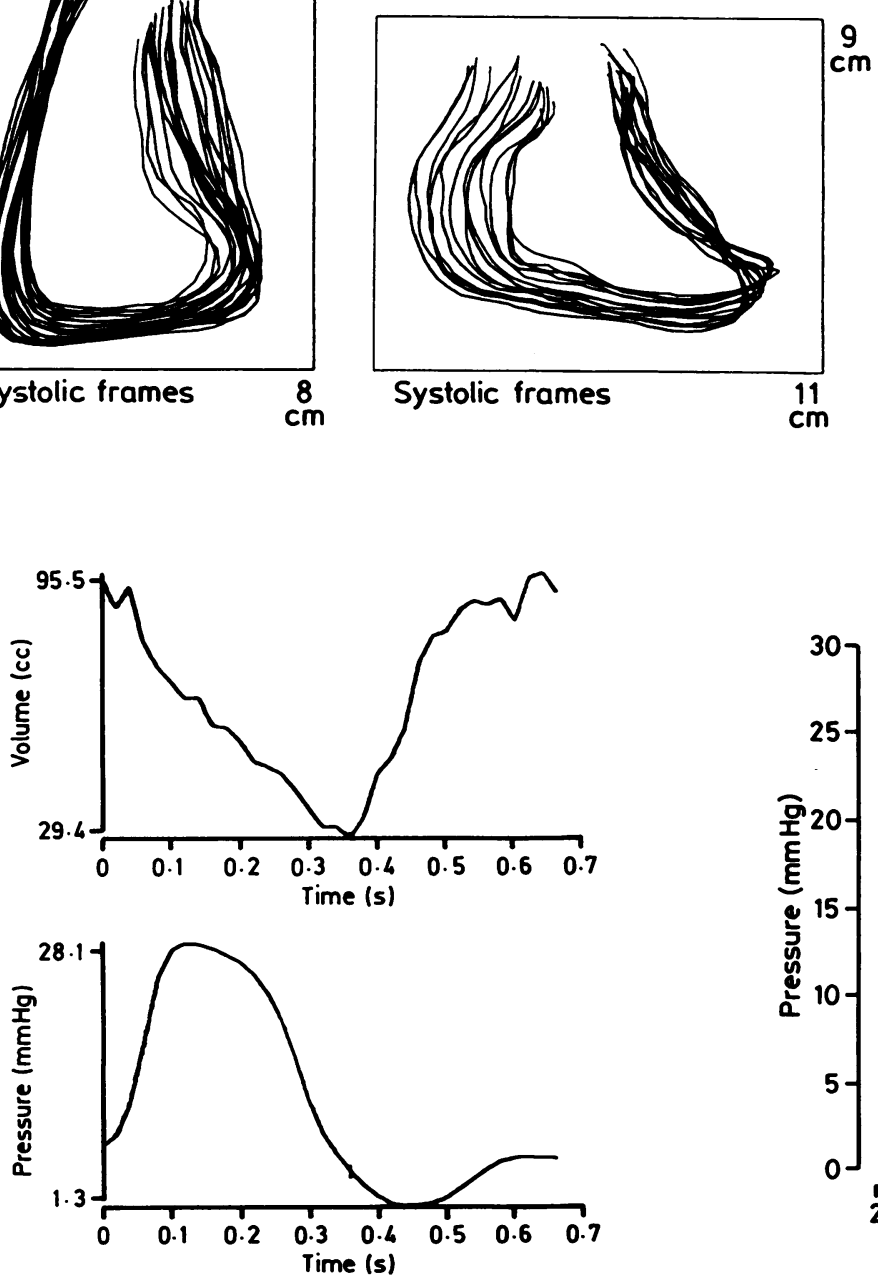

Fig 3 Plots showing simultaneous right ventricular pressure and volume curves.

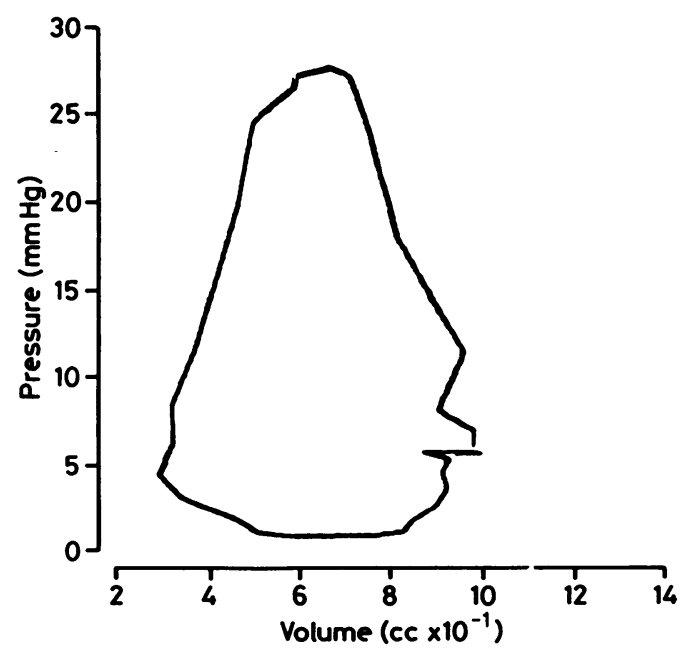

Fig 4 Normal right ventricular pressure-volume loop derived from the curves shown in fig 3. 


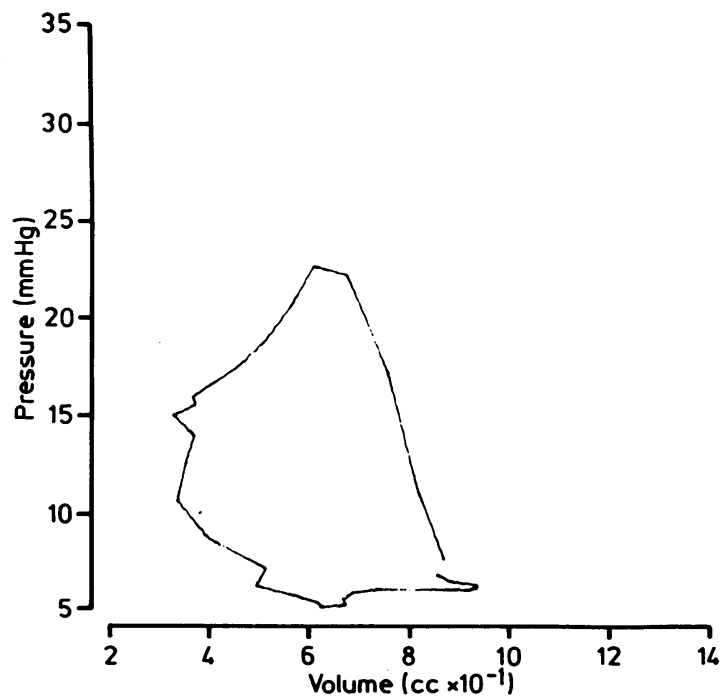

Fig 5 Normal right ventricular pressure-volume loop. In this example there is a better defined limb of isovolumic relaxation. Maximum work $944.8 \mathrm{~mm} \mathrm{Hg} \times \mathrm{ml}$ and efficiency $62 \%$.

measured by a method previously described for the left ventricle ${ }^{12}$ and expressed as the ratio of the area enclosed by the loop to that of a square or rectangle that just encloses it. This represents the ratio of actual work done to the maximum possible performed by a ventricle with the same stroke volume and pressure change.

In each case the cycle was digitised on another occasion and where possible $(n=7)$ a second cycle was digitised to assess intraobserver and beat to beat variation.

STATISTICAL ANALYSIS

Group data were expressed as mean (1 SD). The actual cast volumes, measured by fluid displacement, were compared with volumes calculated by linear regression analysis by the method of least squares.

In the clinical studies intraobserver and beat to beat variability of ventricular volumes and ejection fraction were expressed as the mean percentage difference and as a coefficient of repeatability. ${ }^{13}$ Assuming that the mean difference between the two sets of observations is zero (that is there is no systematic over or underestimation) and that the difference is unrelated to the absolute size of the measurement (for example the error does not increase with the size of the ventricle), this coefficient is equal to two standard deviations of the mean absolute difference, and is thus expressed in the same units as the original measurements.

\section{Results}

\section{CAST DATA}

The volume of the casts ranged from 10 to $91 \mathrm{ml}$. Fig $6 a$ and $b$ shows the relation between cast volume measured by fluid displacement and volume derived from the two pairs of orthogonal views. Excellent correlation coefficients were obtained (both $\mathbf{r}=$ 0.99), with low standard errors and $Y$ intercepts suggesting that the method was applicable to calculation of small volumes. The regression equations for each pair of orthogonal views were: $(i)$ anteroposterior and lateral views: volume (true) $=0.86$ volume (calc) $+0.68(\mathrm{SEE}=4 \mathrm{ml} ; \mathrm{p}<0.001)$; (ii) right and left anterior oblique views: volume (true) $=0.81$ volume (calc) $+0.48($ SEE $=4 \mathrm{ml} ; \mathrm{p}<0.001)$.

\section{PATIENT DATA}

Left heart haemodynamic function and coronary arteries were normal in all patients. Peak right ventricular systolic pressure was 26 (4) $\mathrm{mm} \mathrm{Hg}$, and right ventricular end diastolic pressure was 6 (4) $\mathrm{mm} \mathrm{Hg}$. There was no angiographic evidence of tricuspid regurgitation in any of the patients. Satisfactory right ventriculograms were obtained in all, but because of technical problems such as multiple extrasystoles or poor opacification only a single beat was available for analysis in three of the 10 patients. Calculated right ventricular volumes were corrected by the regression equations shown above and expressed per square metre body surface area. End diastolic volume index was $64(13) \mathrm{ml} / \mathrm{m}^{2}$ and stroke volume index was $43(8) \mathrm{ml} / \mathrm{m}^{2}$. The mean right ventricular ejection fraction for the group was $62(6) \%$.

\section{RIGHT VENTRICULAR PRESSURE-VOLUME}

\section{RELATIONS}

Right ventricular pressure-volume loops tended to be more triangular than the typical left ventricular pressure-volume loop (fig 7). Ejection from the ventricle began early during pressure upstroke with a mean of $39(4 \%)$ of the stroke volume being ejected before peak right ventricular pressure. Consequently there was a poorly defined phase of isovolumic contraction. Interestingly ejection continued well beyond the development of peak right ventricular pressure with $43(9) \%$ of the stroke volume being ejected during pressure fall. In only five patients was there a well defined end systolic shoulder to the loop (fig 5).

Right ventricular systolic stroke work was 0.078 $+0.021 \mathrm{~J} / \mathrm{m}^{2}$ per beat and the resulting cycle efficiency was $56(8) \%$ (normal for left ventricle = $72-84 \%$ ). 

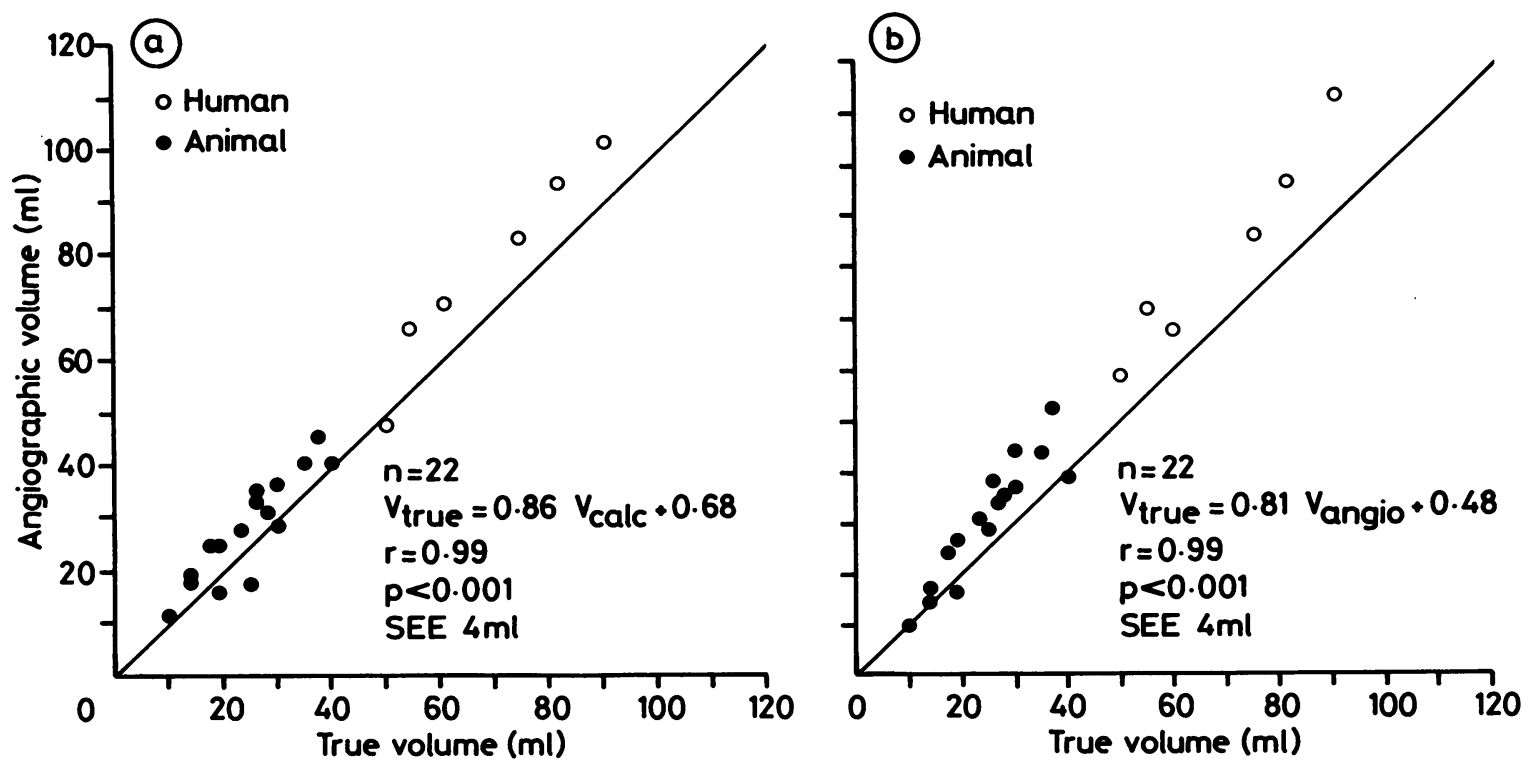

Fig 6 Graphs showing the relation between right ventricular cast volumes measured by fluid displacement and from biplane angiograms. (a) Anteroposterior and lateral views. (b) $30^{\circ}$ right anterior oblique and $60^{\circ}$ left anterior oblique views.

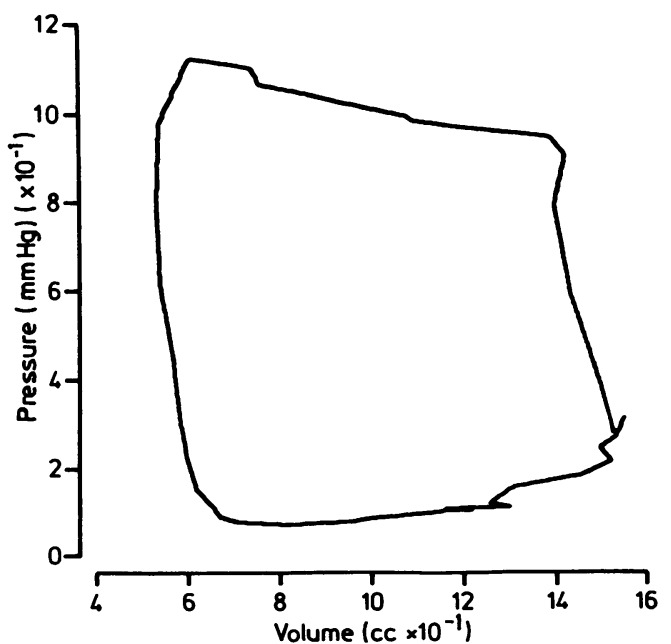

Fig 7 Typical example of a normal left ventricular pressure-volume loop.

INTRAOBSERVER AND BEAT TO BEAT REPRODUCIBIL I T Y

The table shows intraobserver and beat to beat repeatability.

\section{Discussion}

Investigation of left ventricular pressure-volume relations has greatly increased our understanding of
Table Intraobserver and beat to beat reproducibility of measured variables

\begin{tabular}{lrrr}
\hline & $E D V$ & $S V$ & $R V E F$ \\
\hline Intraobserver: & & & \\
Mean \% difference & 5.9 & 6.8 & 5.9 \\
Coefficient of repeatability & $5 \cdot 2$ & 3.6 & 4.0 \\
Beat to beat (n = 7): & & & \\
Mean \% difference & 11.2 & 13.5 & 12.9 \\
Coefficient of repeatability & 9.2 & 9.8 & 14.0 \\
\hline
\end{tabular}

EDV, end diastolic volume; SV, stroke volume; RVEF, right ventricular ejection fraction.

left ventricular function and it is perhaps surprising, therefore, that there is so little information on right ventricular pressure-volume relations. One explanation is the difficulty of accurately measuring right ventricular volume because of its complex geometry. Nevertheless, several attempts have been made. ${ }^{135}$ Although methods based on area-length derivations and geometric models ${ }^{1-3}$ accurately measure the volumes of casts of the right ventricle, they may be inappropriate for the measurement of right ventricular volume throughout the cardiac cycle because $\stackrel{\oplus}{?}$ changes in cavity shape and spatial orientation during contraction and relaxation, and under the abnormal conditions of chronic pressure or volume overload, make it unlikely that a single geometric model can be applied in all circumstances.

Methods using Simpson's rule go some way towards overcoming these difficulties but certain 
geometric assumptions are inherent in any method that attempts to measure ventricular volume from angiograms. In this study we have assumed that each slice of the right ventricle is elliptical and when we digitised both the casts and ventriculograms we included papillary muscles and trabeculae in the cavity outline. It is not surprising, therefore, that our method overestimates ventricular volumes when compared with those derived from fluid displacement of the casts. None the less, this overestimation appears to be consistent and, by applying the appropriate regression equation, right ventricular volume can be estimated with a degree of accuracy approaching that previously described for the left ventricle. ${ }^{14}$ Indeed simple measurements of end diastolic and end systolic volumes in our patients were similar to those reported by others ${ }^{515}$ and the derived stroke volume index $\left(43(8) \mathrm{ml} / \mathrm{m}^{2}\right)$ is similar to that reported for the left ventricle. ${ }^{16}$ Thus it should be possible to apply our technique to frame by frame analysis of right ventricular volume in a manner similar to that previously performed for the left ventricle.

Gated blood pool radionuclide angiography has been used to assess right ventricular pressurevolume relations ${ }^{9}$ but such techniques calculate volume changes by averaging data obtained from many cardiac cycles and hence pressure and volume measurements can never be truly simultaneous. Furthermore, the relatively low sampling rate (30 frames per second) and variations in the $R R$ interval during data acquisition introduce additional errors. In our study right ventricular volume was calculated every $20 \mathrm{~ms}$ and close synchronisation of pressure and volume was achieved. Beat to beat variability and intraobserver variability of measured indices were acceptable and the overall shape of the right ventricular pressure-volume loops was both reproducible and similar in all patients.

The very different loading conditions of the right and left ventricles might be expected to influence their relative pressure-volume characteristics and indeed this has been suggested by the study of crosscirculated canine right ventricles. ${ }^{8}$ The authors of this report commented on the triangular shape of the right ventricular pressure-volume loop resulting from the continued fall in cavity volume after peak right ventricular pressure. Our study confirms these findings in human beings. Unlike the square or rectangular loop of the normal left ventricle (fig 7) in which there is little change in cavity volume during the two isovolumic periods and in which there is a well defined end systolic shoulder, we have shown that ejection from the right ventricle began during the pressure upstroke and consequently a phase of isovolumic contraction was difficult to define. The observation that ejection continued while right ventricular pressure was falling was a striking feature in our study; as much as $60 \%$ of the stroke volume was ejected after peak systolic pressure. In only five patients was there a well-defined end systolic shoulder to the pressure-volume loop.

That the right ventricle can continue to eject during a fall in pressure was suggested indirectly from observations by Shaver et al in 1974 who, using simultaneous right ventricular and pulmonary artery pressure measurements, described a so-called "hangout period" of up to $90 \mathrm{~ms}$ between right ventricular pressure fall and pulmonary valve closure in patients with normal right heart pressures. ${ }^{17}$ The ability of the right ventricle to eject blood as cavity pressure is falling presumably reflects the low hydraulic impedance to flow and high capacitance of the normal pulmonary vascular bed since this hangout period was greatly reduced when pulmonary vascular resistance was raised. There are no human right ventricular pressure-volume data on the effect of pressure loading, but further support for this phenomenon being a load-dependent one, rather than an intrinsic property of the right ventricular myocardium, comes from similar appearances of pressure-volume loops obtained from left ventricles ejecting against the much reduced afterloads that are present after the Mustard operation (unpublished observations) and during treatment with vasodilators. ${ }^{18}$

The external mechanical work performed by the right ventricle on the circulation during systole is equal to the area enclosed by the pressure-volume loop. ${ }^{19}$ The ratio of this area to the area of a square or rectangle that just encloses it represents the ratio of actual work done to the maximum possible done by a ventricle with the same pressure and volume changes and has been termed "cycle efficiency". Despite the finding that the work performed by the right ventricle in ejecting its stroke volume is considerably lower than that for the left ventricle, its "efficiency", as measured by this index, is low (58 $(6) \%)$. Cycle efficiency is, however, simply a measure of the ability of the ventricle to act as a square wave pump and the low cycle efficiency observed for the normal right ventricle should not be interpreted as a reflection of efficiency in terms of its energetics, but rather as a convenient method of quantifying the departure of the shape of its pressure-volume loop from that of a square or rectangle.

Indeed there is evidence that the right ventricle may be more efficient than the left in terms of its energetics. It has been demonstrated previously that a considerable amount of potential energy stored in the left ventricular wall at end systole is dissipated in isovolumic relaxation. ${ }^{20}$ In experimental prepara- 
tions much of this potential energy can be converted to external mechanical work by manipulating the afterload against which the left ventricle ejects. ${ }^{20}$ Left ventricular pressure-volume loops under these circumstances are similar in shape to those we have recorded for the normal right ventricle and this suggests that the normal right ventricle may be converting more of the potential energy stored in its walls at maximum pressure into external mechanical work.

Further studies are required to evaluate the effect of loading conditions on the human right ventricular pressure-volume relation and until then the clinical implications of our results are likely to be speculative. Our observations do suggest, however, that previous measurements of isovolumic and end systolic indices of right ventricular contractility should be interpreted with caution.

We have shown that it is possible to measure right ventricular volume throughout the cardiac cycle and construct pressure-volume loops. Analysis of these loops showed fundamental differences between the pressure-volume relation of the normal left and right ventricles, which presumably reflect the low hydraulic impedance to flow in the pulmonary circulation.

We thank Dr Derek Gibson, Susan Jones, and David Hughes for help in undertaking and preparing for this study.

ANR is holder of a British Heart Foundation grant.

\section{References}

1 Shimazaki Y, Kawashima Y, Mori T, Beppu S, Yokota $K$. Angiographic volume estimation of right ventricle. Chest 1980;77:390-5.

2 Graham TP, Jarkamani JM, Atwood GF, Canent RV. Right ventricular volume determinations in children. Circulation 1973;47:144-53.

3 Ferlinz J, Gorlin R, Cohn PF, Herman MV. Right ventricular performance in patients with coronary artery disease. Circulation 1975;52:608-15.

4 Gentzler RD, Briselli MF, Gault JH. Angiographic estimation of right ventricular volume in man. Circulation 1974;50:324-30.

5 Fisher EA, DuBrow IW, Hastreiter AR. Right ventric- ular volume in congenital heart disease. Am J Cardiol 1975;36:67-75.

6 Ferlinz J. Right ventricular performance in adult cardiovascular disease. Prog Cardiovasc Dis 1982;25:225-67.

7 Baker EJ, Shubao C, Clarke SE, Fogelman I, Maisey MN, Tynan M. Radionuclide measurement of right ventricular function in atrial septal defect, ventricular septal defect, and complete transposition of the great arteries. Am J Cardiol 1986;57:1142-6.

8 Maughan WL, Shoukas AA, Sagawa K, Weisfeldt ML. Instantaneous pressure-volume relationship of the canine right ventricle. Circ Res 1979;44:309-15.

9 Friedman BJ, Lozner EC, Curfman GD, Herzberg D, Rolett EL. Characterisation of the human right ventricular pressure-volume relation: effect of dobutamine and right coronary artery stenosis. $\mathrm{J} \mathrm{Am}$ Coll Cardiol 1984;4:999-1005.

10 Pridie RB, Parnell B. The importance of magnification in left ventriculography. $B r J$ Radiol 1980;53:642-6.

11 Yettram AL, Vinson CA, Gibson DG. Computer modelling of the human left ventricle. $J$ Biomech 1982;104:148-52.

12 Gibson DG, Brown DJ. Assessment of left ventricular systolic function in man from simultaneous echocardiographic and pressure measurements. $\mathrm{Br}$ Heart $J$ 1976;38:8-17.

13 Bland JM, Altman DG. Statistical methods for assessing agreement between two methods of clinical measurement. Lancet 1986;i:307-10.

14 Starling MR, Walsh RA. Accuracy of biplane axial oblique and oblique cineangiographic left ventricular cast volume determinations using a modification of Simpson's rule algorithm. Am Heart J 1985;110: 1219-25.

15 Ferlinz J. Right ventricular performance in essential hypertension. Circulation 1980;61:156-62.

16 Nicholls AB, Pearson MH, Sciacca RR, Cannon PJ. Ventricular efficiency in coronary artery disease. J Am Coll Cardiol 1986;7:270-9.

17 Shaver JA, Nadolny RA, O'Toole JD, et al. Sound pressure correlates of the second heart sound. Circulation 1974;49:316-25.

18 Weber KT, Janicki JS. Instantaneous force-velocitylength relations in isolated dog heart. Am J Physiol (H) 1977;232:241-9.

19 Suga H, Yamada O, Goto Y. Energetics of ventricular contraction as traced in the pressure-volume diagram. Fed Proc 1984;43:2411-3.

20 Suga $H$. External mechanical work from relaxing ventricle. Am J Physiol (H) 1979;236:494-7. 